\title{
Adaptive Neural Gradient Descent Control for a Class of Nonlinear Dynamic Systems with Chaotic Phenomenon
}

\author{
Xiujuan Liu ${ }^{1}$ and Tian $\operatorname{Lan}^{2}$ \\ ${ }^{1}$ College of Science, Liaoning University of Technology, Jinzhou, Liaoning 121001, China \\ ${ }^{2}$ School of Advanced Engineering, Beihang University, Beijing 100191, China \\ Correspondence should be addressed to Tian Lan; lantianjz@yeah.net
}

Received 17 October 2014; Accepted 13 November 2014

Academic Editor: Qing Gao

Copyright (c) 2015 X. Liu and T. Lan. This is an open access article distributed under the Creative Commons Attribution License, which permits unrestricted use, distribution, and reproduction in any medium, provided the original work is properly cited.

\begin{abstract}
A neural network controller design is studied for a class of nonlinear chaotic systems with uncertain parameters. Because the chaos phenomena are often in this class of systems, it is indispensable to control this class of systems. At the same time, due to the presence of uncertainties in the chaotic systems, it results in the difficulties of the controller design. The neural networks are employed to estimate the uncertainties of the systems and a controller is designed to overcome the chaos phenomena. The main contribution of this paper is that the adaptation law can be determined via the gradient descent algorithm to minimize a cost function of error. It can prove the stability of the closed-loop system. The numerical simulation is specified to pinpoint the validation of the approach.
\end{abstract}

\section{Introduction}

The control problem for complex and ill-defined systems is a very significant work in practice. The uncertainties, unknown parameters, and unmodeled dynamics, and so forth, belong to ill-defined systems. There are many practical plants that are ill-defined, such as robot, chemical systems, underactuated systems, and chaotic systems, and their stability problems are hard to be solved. A good way for solving ill-defined systems is to use the neural network control scheme and this scheme has been used to perform important task in practice. Simultaneously, a lot of achievements have been gained for some different classes of ill-defined systems. In [19], the controller tracking designs were solved for ill-defined continuous-time systems which contain uncertainties and nonlinear property. For ill-defined discrete-time systems, several design strategies were given in [10-13].

Recently, the stability of chaotic systems, which belong to a class of ill-defined systems, has been an active research direction. In [14], the control problem for chaotic systems was studied. The functions of chaotic systems are nonlinear and do not require satisfying Lipschitz condition. A synchronization sliding mode control algorithm was proposed in [15] for two classes of chaotic systems based on RBF neural networks. A combined disturbance observer was proposed and the update law of uncertain parameters was shown to monitor the combined disturbance. A function projective synchronization for fractional order chaotic systems with unknown parameters was investigated in [16] by using adaptive control modified method. In [17], a novel synchronization modified function projective scheme for identical and nonidentical hyperchaotic complex chaotic systems with uncertain parameters was developed. An adaptive chatterfree control via sliding mode design was pointed out in [18] for uncertain chaotic system without the knowledge of the bounds of the uncertain term representing the model uncertainty and unknown disturbance. In [19], the time stability for a general chaotic system was analyzed by utilizing adaptive feedback terminal sliding mode control. However, the adaptation laws of these approaches are obtained without using gradient descent method. A main advantage of gradient descent method is that it can minimize a cost function of error. This paper will use gradient descent method to control a class of chaotic systems.

Based on the above presentations, an adaptive control algorithm via the neural networks is investigated for a class of chaotic systems. By using the neural network approximation, the unknown function is modeled and chaos phenomena can 
be solved by using a stable controller. Adaptive law design is proposed via the gradient descent algorithm to minimize a cost function of error. The Lyapunov analysis is employed to prove the stability of the closed-loop system and a simulation is to validate the effectiveness of the approach.

\section{Problem and Formulation}

Consider the chaotic systems as the following form:

$$
\begin{aligned}
& \dot{x}_{1}=x_{2}, \\
& \dot{x}_{2}=x_{3}, \\
& \dot{x}_{3}=\alpha_{1} x_{1}+\alpha_{2} x_{2}+\alpha_{3} x_{3}+\alpha_{4} x_{1}^{3},
\end{aligned}
$$

where $\alpha_{i}, i=1, \ldots, 4$, are unknown parameters. The system (1) is also called Arneodo chaotic system. When there does not exist a control input, the chaotic system is unstable.

Let $f(\bar{x})=\bar{\alpha} \bar{x}^{T}$ where $\bar{\alpha}=\left[\alpha_{1}, \alpha_{2}, \alpha_{3}, \alpha_{4}\right]$ and $\bar{x}=$ $\left[x_{1}, x_{2}, x_{3}, x_{1}^{3}\right]^{T}$. Then, one has

$$
\begin{aligned}
& \dot{x}_{1}=x_{2}, \\
& \dot{x}_{2}=x_{3}, \\
& \dot{x}_{3}=f(\bar{x})+u,
\end{aligned}
$$

where $\bar{x}=\left[x_{1}, x_{2}, x_{3}\right]^{T} \in R^{3}$ is the state vector of the system and $u \in R$ is the scalar control input.

In this study, the control objective is to design an adaptive neural control algorithm so that the system is stable; that is, all the signals in the closed-loop system are bounded.

The error vector is defined as

$$
\bar{z}=[z, \dot{z}, \ddot{z}]^{T} \in R^{3}
$$

where

$$
z=x_{1}
$$

Then, we know that

$$
z^{(3)}=f(\bar{x})+u
$$

It can be written as follows:

$$
\dot{\bar{z}}=A_{0} \bar{z}+B[f(\bar{x})+u]
$$

where

$$
A_{0}=\left[\begin{array}{lll}
0 & 1 & 0 \\
0 & 0 & 1 \\
0 & 0 & 0
\end{array}\right] \in R^{3 \times 3}, \quad B=\left[\begin{array}{l}
0 \\
0 \\
1
\end{array}\right] \in R^{3} .
$$

We select $k=\left[k_{0}, k_{1}, k_{2}\right]^{T}$ to ensure that $A=A_{0}-B k^{T}$ is stable. Therefore, there exists a matrix $Q$ such that

$$
A^{T} P+P A=-Q
$$

where $P$ and $Q$ are positive definite symmetric matrices.
Define $c$ as

$$
c=k^{T} \bar{z}+\beta \tanh \left(\frac{B^{T} P \bar{z}}{\varepsilon}\right),
$$

where $\beta>0$ and $\varepsilon>0$ are the constants.

By adding and subtracting the term $c$ to (6), we know that

$$
\dot{\bar{z}}=A \bar{z}-B \beta \tanh \left(\frac{B^{T} P \bar{z}}{\varepsilon}\right)+B[f(\bar{x})+u-c] .
$$

Based on the implicit function theorem, there exists an ideal controller $u^{*}(x, c)$ so that

$$
f(\bar{x})+u^{*}(\bar{x}, c)-c=0
$$

If $u$ is selected as $u=u^{*},(10)$ is simplified as

$$
\dot{\bar{z}}=A \bar{z}-B \beta \tanh \left(\frac{B^{T} P \bar{z}}{\varepsilon}\right) .
$$

Consider the following Lyapunov function:

$$
V=\bar{z}^{T} P \bar{z} \text {. }
$$

Combining with (12) and (8), the time derivative of (13) can be written as

$$
\begin{aligned}
\dot{V}= & {\left[\bar{z}^{T} A^{T}-B^{T} \beta \tanh \left(\frac{B^{T} \bar{z}}{\varepsilon}\right)\right] P \bar{z} } \\
& +\bar{z}^{T} P A \bar{z}-\bar{z}^{T} P B \beta \tanh \left(\frac{B^{T} \bar{z}}{\varepsilon}\right) \\
= & \bar{z}^{T}\left(A^{T} P+P A\right) \bar{z}-B^{T} \beta \tanh \left(\frac{B^{T} \bar{z}}{\varepsilon}\right) P \bar{z} \\
& -\bar{z}^{T} P B \beta \tanh \left(\frac{B^{T} \bar{z}}{\varepsilon}\right) \\
= & -\bar{z}^{T} Q \bar{z}-2 \beta B^{T} P \bar{z} \tanh \left(\frac{B^{T} \bar{z}}{\varepsilon}\right) .
\end{aligned}
$$

Because $B^{T} P \bar{z} \tanh \left(B^{T} P \bar{z} / \varepsilon\right)>0, \dot{V}<0$. Then, the system is stable. Nevertheless, $u^{*}(\bar{x}, c)$ is not effective owing to unknown parameters.

\section{Gradient Descent Controller Design}

In Section 2, we assume that there is $u^{*}(\bar{x}, c)$ can realize the objective. We can determine the presence of the ideal controller with the aid of implicit function theorem, but we cannot get the way to build it. In this section, we use adaptive neural system to determine the controller.

$u^{*}(\bar{x}, c)$ is approximated as follows:

$$
u^{*}(\bar{\xi})=\bar{w}^{T}(\bar{\xi}) \theta^{*}+\delta(\bar{\xi})
$$

where $\bar{\xi}=\left[\bar{x}^{T}, c\right]^{T}$ and $\delta(\xi)$ is the fuzzy approximation error, $\theta^{*}$ is an ideal parameter vector, and $\bar{w}(\bar{\xi})$ is a basic function 
vector. In this study, the approximation error is bounded; that is, $|\delta(\bar{\xi})| \leq \bar{\delta}$ with $\bar{\delta}>0$ being a constant.

$\theta^{*}$ is unknown; then, choose $\theta$ as an estimate of $\theta^{*}$. Define the control law as

$$
u(\bar{\xi})=\bar{w}^{T}(\bar{\xi}) \theta .
$$

We define the error between $u$ and $u^{*}$ as follows:

$$
z_{u}=u^{*}-u \text {. }
$$

By using (15) and (16), (17) can be written as

$$
\begin{aligned}
z_{u} & =u^{*}-u \\
& =\bar{w}^{T}(\bar{\xi}) \theta^{*}+\delta(\bar{\xi})-\bar{w}^{T}(\bar{\xi}) \theta \\
& =\bar{w}^{T}(\bar{\xi}) \tilde{\theta}+\delta(\bar{\xi}),
\end{aligned}
$$

where $\widetilde{\theta}=\theta^{*}-\theta$.

According to (18), $f(\bar{x})+u$ can be expressed as

$$
f(\bar{x})+u=f(\bar{x})+u^{*}-z_{u} .
$$

Substituting (19) into (10) yields

$$
\dot{\bar{z}}=A \bar{z}-B \beta \tanh \left(\frac{B^{T} P \bar{z}}{\varepsilon}\right)+B\left[f(\bar{x})+u^{*}-z_{u}-c\right] .
$$

Substituting (11) into (20),

$$
\dot{\bar{z}}=A \bar{z}-B \beta \tanh \left(\frac{B^{T} P \bar{z}}{\varepsilon}\right)-B z_{u} .
$$

Then, we have

$$
z^{(3)}+k^{T} \bar{z}+\beta \tanh \left(\frac{B^{T} P \bar{z}}{\varepsilon}\right)=z_{u} .
$$

Then, consider a quadratic cost function, which is used to measure the discrepancy between the ideal and the neural controller. And the function can be defined as

$$
J(\theta)=\frac{1}{2} z_{u}^{2}=\frac{1}{2}\left(u^{*}-u\right)^{2}=\frac{1}{2}\left(u^{*}-\bar{w}^{T}(\bar{\xi}) \theta\right)^{2} .
$$

Here, we will minimize (23) based on the gradient descent algorithm. We obtain the following first-order differential equation

$$
\dot{\theta}=-\gamma(t) \nabla_{\theta} J(\theta),
$$

where $\gamma(t)$ is a positive parameter.

By employing (23), it has

$$
\frac{\partial J(\theta)}{\partial \theta}=-\bar{w}(\bar{\xi}) z_{u}
$$

Hence, the gradient descent algorithm can be represented as

$$
\dot{\theta}=\lambda(t) \bar{w}(\bar{\xi}) z_{u} .
$$

Because $z_{u}$ is unavailable, we need to make (26) calculable; we select $\gamma(t)=\gamma_{0}$ where $\gamma_{0}>0$. Then, (26) can be redefined as

$$
\dot{\theta}=\gamma_{0} \bar{w}(\bar{\xi}) z_{u} .
$$

By substituting (22) into (27), we know that

$$
\dot{\theta}=\lambda_{0} \bar{w}(\bar{\xi})\left\{z^{(3)}+\bar{k}^{T} \bar{z}+\beta \tanh \left(\frac{\bar{b}^{T} P \bar{z}}{\varepsilon}\right)\right\} .
$$

In order to improve the robustness of (27), we modify it as follows:

$$
\dot{\theta}=\gamma_{0} \bar{w}(\bar{\xi})\left\{z^{(3)}+k^{T} \bar{z}+\beta \tanh \left(\frac{B^{T} P \bar{z}}{\varepsilon}\right)\right\}-\gamma_{0} \sigma \theta,
$$

where $\sigma>0$ is a small constant.

Then, we consider the following function:

$$
V_{\theta}=\frac{1}{2 \gamma_{0}} \widetilde{\theta}^{T} \widetilde{\theta} .
$$

Considering (22) and (29), the time derivative of (29) is

$$
\begin{aligned}
\dot{V}_{\theta} & =-\frac{1}{\gamma_{0}} \widetilde{\theta}^{T} \dot{\theta}=-\frac{1}{\gamma_{0}} \widetilde{\theta}^{T}\left(\gamma_{0} \bar{w}(\bar{\xi}) z_{u}-\gamma_{0} \sigma \theta\right) \\
& =-\widetilde{\theta}^{T}\left(\bar{w}(\bar{\xi}) z_{u}-\sigma \theta\right) .
\end{aligned}
$$

From (18), we get

$$
\bar{w}^{T}(\bar{\xi}) \widetilde{\theta}=z_{u}-\delta(\bar{\xi}) .
$$

By substituting (32) into (31), (31) becomes

$$
\dot{V}_{\theta}=-z_{u}^{2}+\delta(\bar{\xi}) z_{u}+\sigma \widetilde{\theta}^{T} \theta .
$$

Consider the following inequalities:

$$
\begin{aligned}
\sigma \widetilde{\theta}^{T} \theta= & \frac{\sigma}{2}\|\widetilde{\theta}+\theta\|^{2}-\frac{\sigma}{2}\|\widetilde{\theta}\|^{2}-\frac{\sigma}{2}\|\theta\|^{2} \\
= & \frac{\sigma}{2}\left\|\theta^{*}\right\|^{2}-\frac{\sigma}{2}\|\widetilde{\theta}\|^{2}-\frac{\sigma}{2}\|\theta\|^{2} \\
\leq & \frac{\sigma}{2}\left\|\theta^{*}\right\|^{2}-\frac{\sigma}{2}\|\widetilde{\theta}\|^{2} \\
-z_{u}^{2}+\delta(\bar{\xi}) z_{u}= & -\frac{1}{2} z_{u}^{2}-\frac{1}{2} z_{u}^{2}+\frac{1}{2} \delta(\bar{\xi})^{2} \\
& -\frac{1}{2} \delta(\bar{\xi})^{2}+\delta(\bar{\xi}) z_{u} \\
= & -\frac{1}{2} z_{u}^{2}+\frac{1}{2} \delta(\bar{\xi})^{2} \\
& -\left(\frac{1}{2} \delta(\bar{\xi})^{2}-\delta(\bar{\xi}) z_{u}+\frac{1}{2} z_{u}^{2}\right) \\
= & -\frac{1}{2} z_{u}^{2}+\frac{1}{2} \delta(\bar{\xi})^{2}-\frac{1}{2}\left(\delta(\bar{\xi})-z_{u}\right)^{2} \\
\leq & -\frac{1}{2} z_{u}^{2}+\frac{1}{2} \delta(\bar{\xi})^{2} .
\end{aligned}
$$


Equation (33) can be rewritten as

$$
\dot{V}_{\theta} \Longleftarrow \frac{\sigma}{2}\left\|\theta^{*}\right\|^{2}-\frac{\sigma}{2}\|\widetilde{\theta}\|^{2}-\frac{1}{2} z_{u}^{2}+\frac{1}{2} \delta(\bar{\xi})^{2} .
$$

In addition, the parameter $\theta^{*}$ is constant, and $\delta(\bar{\xi})$ is presumed to be bounded, so it will define $\tau$ as

$$
\tau=\frac{\sigma}{2}\left\|\theta^{*}\right\|^{2}+\sup _{t}\left(\frac{1}{2} \delta(\bar{\xi})^{2}\right) .
$$

Then, (35) can be written as

$$
\begin{aligned}
\dot{V}_{\theta} & =\frac{\sigma}{2}\left\|\theta^{*}\right\|^{2}-\frac{\sigma}{2}\|\theta\|^{2}-\frac{1}{2} z_{u}^{2}+\frac{1}{2} \delta(\bar{\xi})^{2} \\
& \leq-\phi V_{\theta}+\tau-\frac{1}{2} z_{u}^{2} \\
& \leq-\phi V_{\theta}+\tau,
\end{aligned}
$$

where $\phi=\sigma \gamma_{0}$.

Theorem 1. The adaptive law (31) can guarantee that

(1) $\tilde{\theta}$ is bounded and converges to the compact set

$$
\Omega_{\theta}=\left\{\widetilde{\theta} \mid\|\widetilde{\theta}\|^{2} \leq \frac{2 \gamma_{0} \tau}{\phi}\right\}
$$

(2) the estimation error $z_{u}$ is bounded.

Proof. Considering (37) we can notice that $V_{\theta} \geq \tau / \mu$, and $\dot{V}_{\theta}<0$; therefore, $V_{\theta}$ and $\tilde{\theta}$ are bounded, as well as $\delta(\bar{\xi}) \in L_{\infty}$ and $w(\bar{\xi}) \in L_{\infty}$, resulting in $z_{u} \in L_{\infty}$.

On the base of (37), we can establish that

$$
\|\widetilde{\theta}(t)\|^{2} \leq\|\tilde{\theta}(0)\|^{2} z^{-\phi t}+\frac{2 \gamma_{0} \tau}{\mu} .
$$

This means that $\tilde{\theta}$ converges to the compact set

$$
\Omega_{\theta}=\left\{\tilde{\theta} \mid\|\widetilde{\theta}\|^{2} \leq \frac{2 \gamma_{0} \tau}{\phi}\right\}
$$

Along with $V_{\theta} \in L_{\infty}$ and $|\delta(\bar{\xi})| \leq \bar{\delta}$, and integrating (35), then we can get

$$
\begin{aligned}
\int_{t}^{t+T} z_{u}^{2} d \gamma \leq & \left(\bar{\delta}^{2}+\sigma\left\|\theta^{*}\right\|^{2}\right) T \\
& +2\left(V_{\theta}(t)-V_{\theta}(t+T)\right) \\
\leq & c_{0}\left(\bar{\delta}^{2}+\sigma\right) T+c_{1}
\end{aligned}
$$

with $c_{0}=\max \left(1,\left\|\theta^{*}\right\|^{2}\right), c_{1}=2 \sup _{t}\left(V_{\theta}(t)-V_{\theta}(t+T)\right)$, and (41) implies that $z_{u}$ is bounded.

In order to analyze the convergence of the errors, let us consider the following Lyapunov function:

$$
V_{z}=\bar{z}^{T} p \bar{z}
$$

Substituting (8) and (20) into (42) and then differentiating it with respect to time,

$$
\dot{V}_{z}=-\bar{z}^{T} Q \bar{z}-2 \beta B^{T} P \bar{z} \tanh \left(\frac{B^{T} \bar{z}}{\varepsilon}\right)+2 B^{T} P \bar{z}\left(u^{*}-u\right) .
$$

Considering (18), (43) becomes

$$
\begin{aligned}
\dot{V}_{z}= & -\bar{z}^{T} Q \bar{z}-2 \beta B^{T} P \bar{z} \tanh \left(\frac{B^{T} \bar{z}}{\varepsilon}\right) \\
& +2 B^{T} P \bar{z}\left(\bar{w}^{T}(\bar{\xi}) \tilde{\theta}+\delta(\bar{\xi})\right) .
\end{aligned}
$$

Considering (39), we know that

$$
\|\widetilde{\theta}(t)\| \leq\|\widetilde{\theta}(0)\| z^{-0.5 \phi t}+\sqrt{\frac{2 \gamma_{0} \tau}{\phi}} .
$$

Using (45) and the fact that $\bar{w}(\bar{\xi})$ and $\delta(\bar{\xi})$ are bounded, we can know that

$$
\left|\bar{w}^{T}(\bar{\xi}) \widetilde{\theta}+\delta(\bar{\xi})\right| \leq \tau_{0} z^{-0.5 \phi t}+\tau_{1},
$$

where $\tau_{0}>0$ and $\tau_{1}>0$ are some constants.

Therefore, (44) can be written as

$$
\begin{aligned}
\dot{V}_{z}= & -\bar{z}^{T} Q \bar{z}-2 \beta B^{T} P \bar{z} \tanh \left(\frac{B^{T} \bar{z}}{\varepsilon}\right) \\
& +2\left|B^{T} P \bar{z}\right|\left(\tau_{0} z^{-0.5 \phi t}+\tau_{1}\right) .
\end{aligned}
$$

By supporting that $\beta$ is chosen large enough so that $\beta \geq \tau_{1}$, and using $-x \tanh (x / \varepsilon)+|x| \leq k \varepsilon$, with $k=0.2785$, (47) becomes

$$
\dot{V}_{z}=-\bar{z}^{T} Q \bar{z}+2\left|B^{T} P \bar{z}\right| \tau_{0} z^{-0.5 \phi t}+2 \tau_{1} k \varepsilon .
$$

Consider the inequality

$$
2\left|B^{T} P \bar{z}\right| \tau_{0} z^{-0.5 \phi t} \leq 0.5 \bar{z}^{2}+2\left\|B^{T} P\right\|^{2} \tau_{0}^{2} z^{-\phi t} .
$$

Equation (48) becomes

$$
\dot{V}_{z} \leq-\left[\rho_{\min }(Q)-0.5\right]\|\bar{z}\|^{2}+2\left|B^{T} P \bar{z}\right| \tau_{0} z^{-\phi t}+2 \tau_{1} k \varepsilon,
$$

where $\rho_{\min }(Q)$ denotes the minimum eigenvalue of the matrix $Q$ and satisfies the condition that $\rho_{\min }(Q)>0.5$. Equation (50) becomes

$$
\dot{V}_{z} \leq-\phi_{z} V_{z}+2\left|B^{T} P \bar{z}\right| \tau_{0} z^{-\phi t}+2 \tau_{1} k \varepsilon
$$

where $\phi_{z}=\left[\rho_{\min }(Q)-0.5\right] / \rho_{\max }(P)$ and $\rho_{\max }(P)$ is the maximum eigenvalue of the matrix $P$.

Theorem 2. Consider (2). Suppose that the approximation error in (18) is bounded. Then the control law can guarantee the boundedness of the signals $\bar{x}$ and $u$ and the convergence of the error to the compact set

$$
\Omega_{z}=\left\{\bar{z} \mid\|\bar{z}\| \leq \sqrt{2 \tau_{1} k \varepsilon\left(\rho_{\min }(P) \phi_{z}\right)}\right\} .
$$




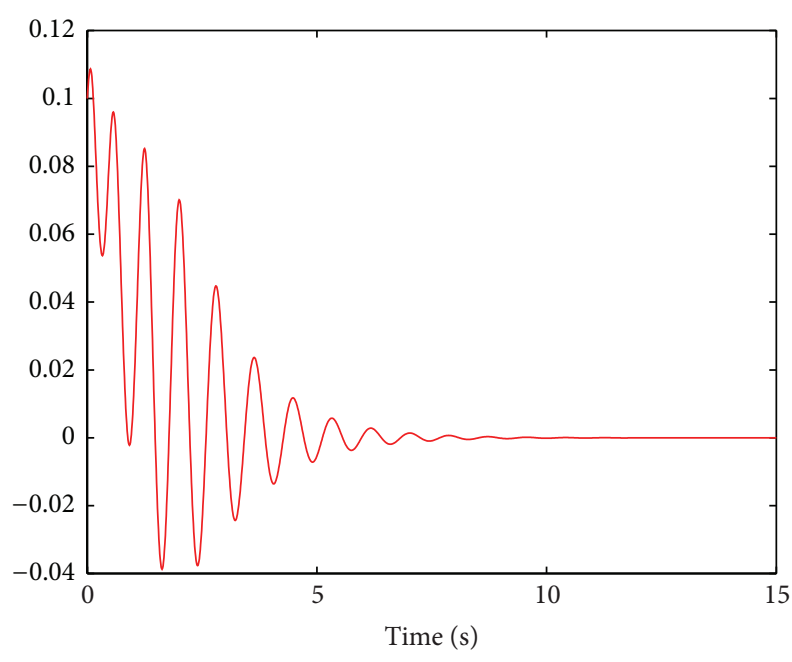

FIGURE 1: The trajectories of $x_{1}$.

Proof. Considering (51) we can notice that, for $\dot{V}_{z}<0$ and $V_{z} \geq\left(2\left|B^{T} P \bar{z}\right| \tau_{0} z^{-\phi t}+2 \tau_{1} k \varepsilon\right) / \phi_{z}$, the error vector $\bar{z}$ is bounded, and the state vector $\bar{x}$ is bounded. Moreover, since the term $2\left|B^{T} P \bar{z}\right| \tau_{0} z^{-\phi t}$ in (51), we can also conclude that the function $V_{z}$ will be asymptotically bounded as

$$
V_{z}<\frac{2 \tau_{1} k \varepsilon}{\phi_{z}}
$$

Thus, the error will converge asymptotically to the compact set

$$
\Omega_{z}=\left\{\bar{z} \mid\|\bar{z}\| \leq \sqrt{2 \tau_{1} k \varepsilon\left(\rho_{\min }(P) \phi_{z}\right)}\right\} .
$$

Based on Theorem 1, we can know that $\tilde{\theta} \in L_{\infty}$; we have that $\theta \in L_{\infty}$, together with $w(\bar{\xi}) \in L_{\infty}$, implies that $u \in L_{\infty}$.

\section{Simulation Results}

In this section, in order to demonstrate the effectiveness of the proposed adaptive neural controller, a system is given in (2) where $\alpha_{1}=5.5, \alpha_{2}=3, \alpha_{3}=-1$, and $\alpha_{4}=-1$.

The purpose is to design an adaptive controller according to (16) which can make all the signals in the system (2) keep bounded. Based on Theorems 1 and 2, we can develop a controller for system (1) satisfying the purpose.

The designed parameters of the proposed control approach are chosen as $\eta=1, \beta=20, \varepsilon=0.02, k=[1,2,1]^{T}$, and $P=\left[\begin{array}{rrr}15 & 5 & 5 \\ 5 & 5 & 5 \\ 5 & 5 & 5\end{array}\right]$. The initial conditions for the system states are supposed as $x_{1}(0)=0.1, x_{2}(0)=0.1, x_{3}(0)=-0.5$, and $\theta(0)=[0.5,0.1,0.2]$.

The simulation results are obtained in Figures 1, 2, 3, 4 , and 5. From Figures 1-3, we get the trajectories of the states $x_{1}, x_{2}, x_{3}$. It means that the states are bounded. Figure 4 illustrates the trajectory of the adaptation law and the action of control input is given in Figure 5. Hence, we can see that the proposed controller makes the closed-loop systems stable.

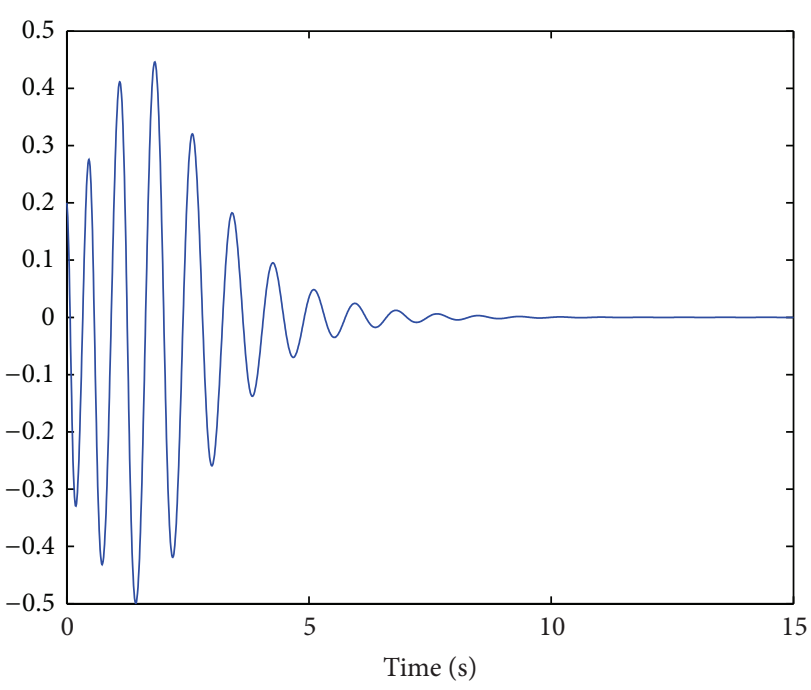

FIgURE 2: The trajectory of $x_{2}$.

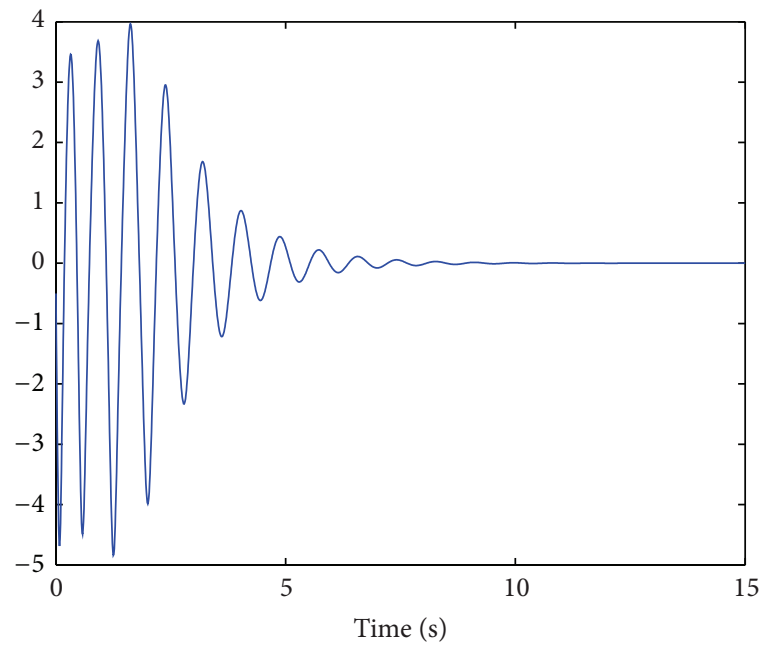

FIGURE 3: The trajectory of $x_{3}$.

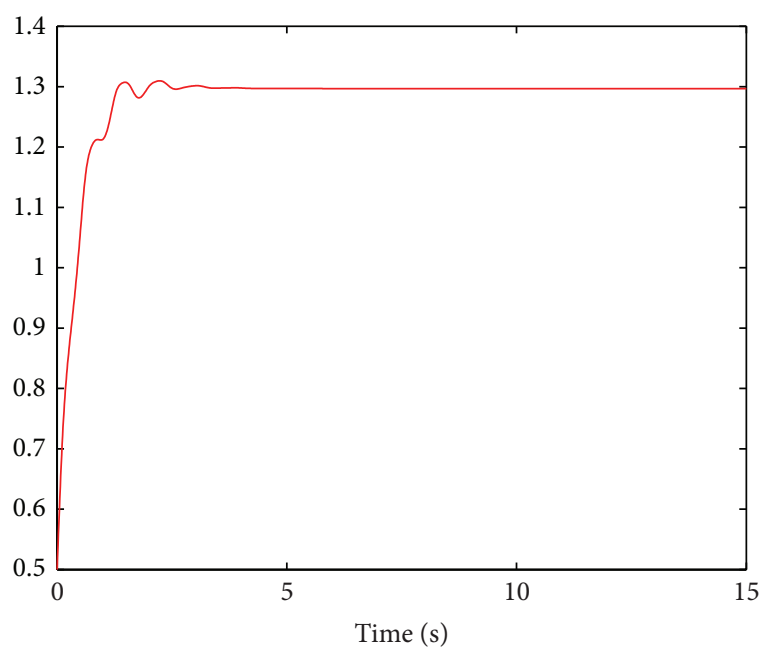

Figure 4: The trajectory of the adaptive law $\theta$. 


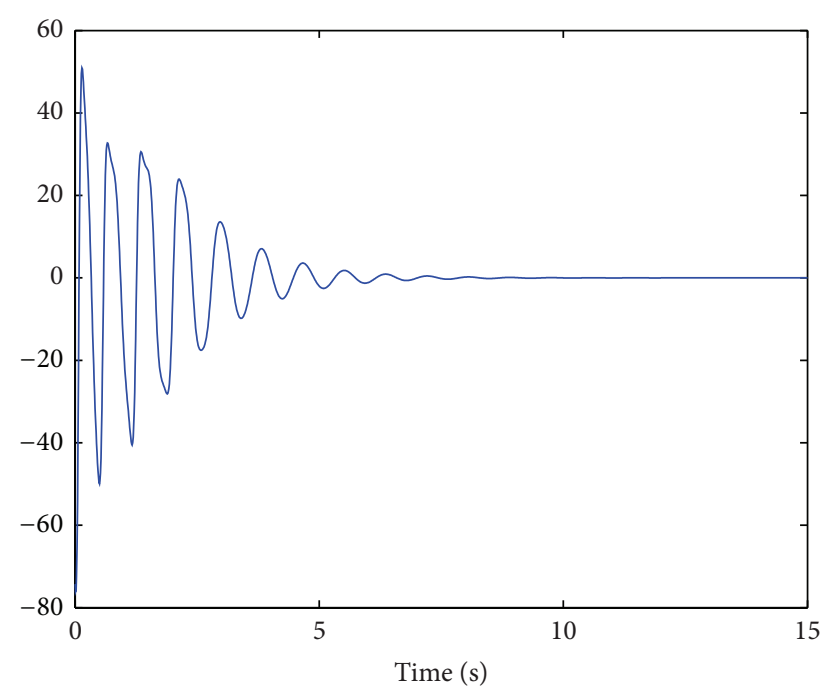

FIGURE 5: The control input $u$.

\section{Conclusion}

In this study, an adaptive control approach for a class of chaotic systems has been developed. The main advantage is to design an adaptive law based on the gradient descent algorithm. This way can minimize a cost function of error. Finally, the theorems and the effectiveness can be validated by Lyapunov analysis and a simulation example. The future research works are to extend the approach to a class of multiinput multioutput systems.

\section{Conflict of Interests}

The authors declare that there is no conflict of interests regarding the publication of this paper.

\section{Acknowledgment}

This work is supported by Natural Science Foundation of Liaoning University of Technology (X201221).

\section{References}

[1] A. J. Calise, N. Hovakimyan, and M. Idan, "Adaptive output feedback control of nonlinear systems using neural networks," Automatica, vol. 37, no. 8, pp. 1201-1211, 2001.

[2] Q. Gao, G. Feng, Y. Wang, and J. Qiu, "Universal fuzzy models and universal fuzzy controllers for stochastic nonaffine nonlinear systems," IEEE Transactions on Fuzzy Systems, vol. 21, no. 2, pp. 328-341, 2013.

[3] C.-M. Lin, A.-B. Ting, C.-F. Hsu, and C.-M. Chung, "Adaptive control for mimo uncertain nonlinear systems using recurrent wavelet neural network," International Journal of Neural Systems, vol. 22, no. 1, pp. 37-50, 2012.

[4] C. L. P. Chen, Y.-J. Liu, and G.-X. Wen, "Fuzzy neural networkbased adaptive control for a class of uncertain nonlinear stochastic systems," IEEE Transactions on Cybernetics, vol. 44, no. 5, pp. 583-593, 2014.
[5] N. Hovakimyan, F. Nardi, A. Calise, and N. Kim, "Adaptive output feedback control of uncertain nonlinear systems using single-hidden-layer neural networks," IEEE Transactions on Neural Networks, vol. 13, no. 6, pp. 1420-1431, 2002.

[6] S. Labiod and T. M. Guerra, "Adaptive fuzzy control of a class of SISO nonaffine nonlinear systems," Fuzzy Sets and Systems, vol. 158, no. 10, pp. 1126-1137, 2007.

[7] C.-W. Chen, P.-C. Chen, and W.-L. Chiang, "Modified intelligent genetic algorithm-based adaptive neural network control for uncertain structural systems," Journal of Vibration and Control, vol. 19, no. 9, pp. 1333-1347, 2013.

[8] C. L. P. Chen, G.-X. Wen, Y.-J. Liu, and F.-Y. Wang, "Adaptive consensus control for a class of nonlinear multiagent time-delay systems using neural networks," IEEE Transactions on Neural Networks and Learning Systems, vol. 25, no. 6, pp. 1217-1226, 2014.

[9] S. Labiod, M. S. Boucherit, and T. M. Guerra, "Adaptive fuzzy control of a class of MIMO nonlinear systems," Fuzzy Sets and Systems, vol. 151, no. 1, pp. 59-77, 2005.

[10] F. C. Chen and H. K. Khalil, "Adaptive control of a class of nonlinear discrete-time systems using neural networks," IEEE Transactions on Automatic Control, vol. 40, no. 5, pp. 791-801, 1995.

[11] Y.-J. Liu, C. L. P. Chen, G.-X. Wen, and S. Tong, "Adaptive neural output feedback tracking control for a class of uncertain discrete-time nonlinear systems," IEEE Transactions on Neural Networks, vol. 22, no. 7, pp. 1162-1167, 2011.

[12] D. Zhang, L. Yu, Q.-G. Wang, and C.-J. Ong, "Estimator design for discrete-time switched neural networks with asynchronous switching and time-varying delay," IEEE Transactions on Neural Networks and Learning Systems, vol. 23, no. 5, pp. 827-834, 2012.

[13] D. Zhang, L. Yu, Q.-G. Wang, C.-J. Ong, and Z.-G. Wu, "Exponential $H_{\infty}$ filtering for discrete-time switched singular systems with time-varying delays," Journal of the Franklin Institute, vol. 349, no. 7, pp. 2323-2342, 2012.

[14] C. C. Hua, X. P. Guan, and P. Shi, "Adaptive feedback control for a class of chaotic systems," Chaos, Solitons \& Fractals, vol. 23, no. 3, pp. 757-765, 2005.

[15] C. Mou, C.-S. Jiang, J. Bin, and Q.-X. Wu, "Sliding mode synchronization controller design with neural network for uncertain chaotic systems," Chaos, Solitons and Fractals, vol. 39, no. 4, pp. 1856-1863, 2009.

[16] S. K. Agrawal and S. Das, "Function projective synchronization between four dimensional chaotic systems with uncertain parameters using modified adaptive control method," Journal of Process Control, vol. 24, no. 5, pp. 517-530, 2014.

[17] L. Chao and W. Xingyuan, "Adaptive modified function projective lag synchronization of hyperchaotic complex systems with fully uncertain parameters," Journal of Vibration and Control, vol. 20, no. 12, pp. 1831-1845, 2014.

[18] X. Zhang, X. Liu, and Q. Zhu, "Adaptive chatter free sliding mode control for a class of uncertain chaotic systems," Applied Mathematics and Computation, vol. 232, pp. 431-435, 2014.

[19] D. Yang and G. Qiu, "Terminal sliding mode control with adaptive feedback control in a class of chaotic systems," Mathematical Problems in Engineering, vol. 2014, Article ID 267024, 6 pages, 2014. 


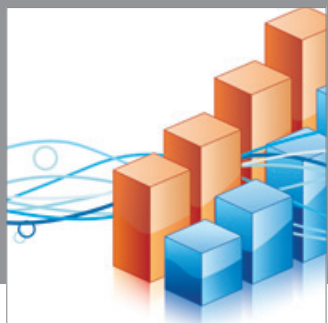

Advances in

Operations Research

mansans

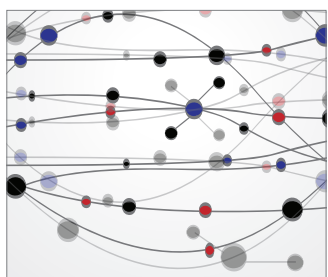

The Scientific World Journal
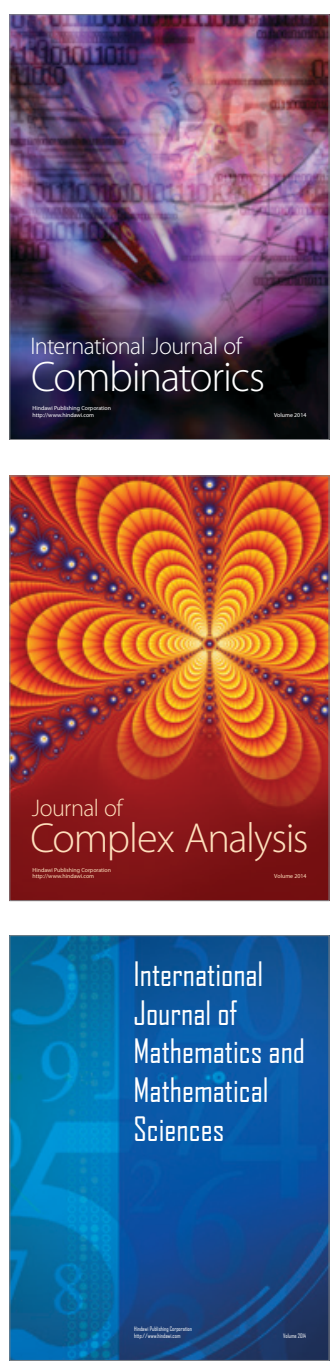
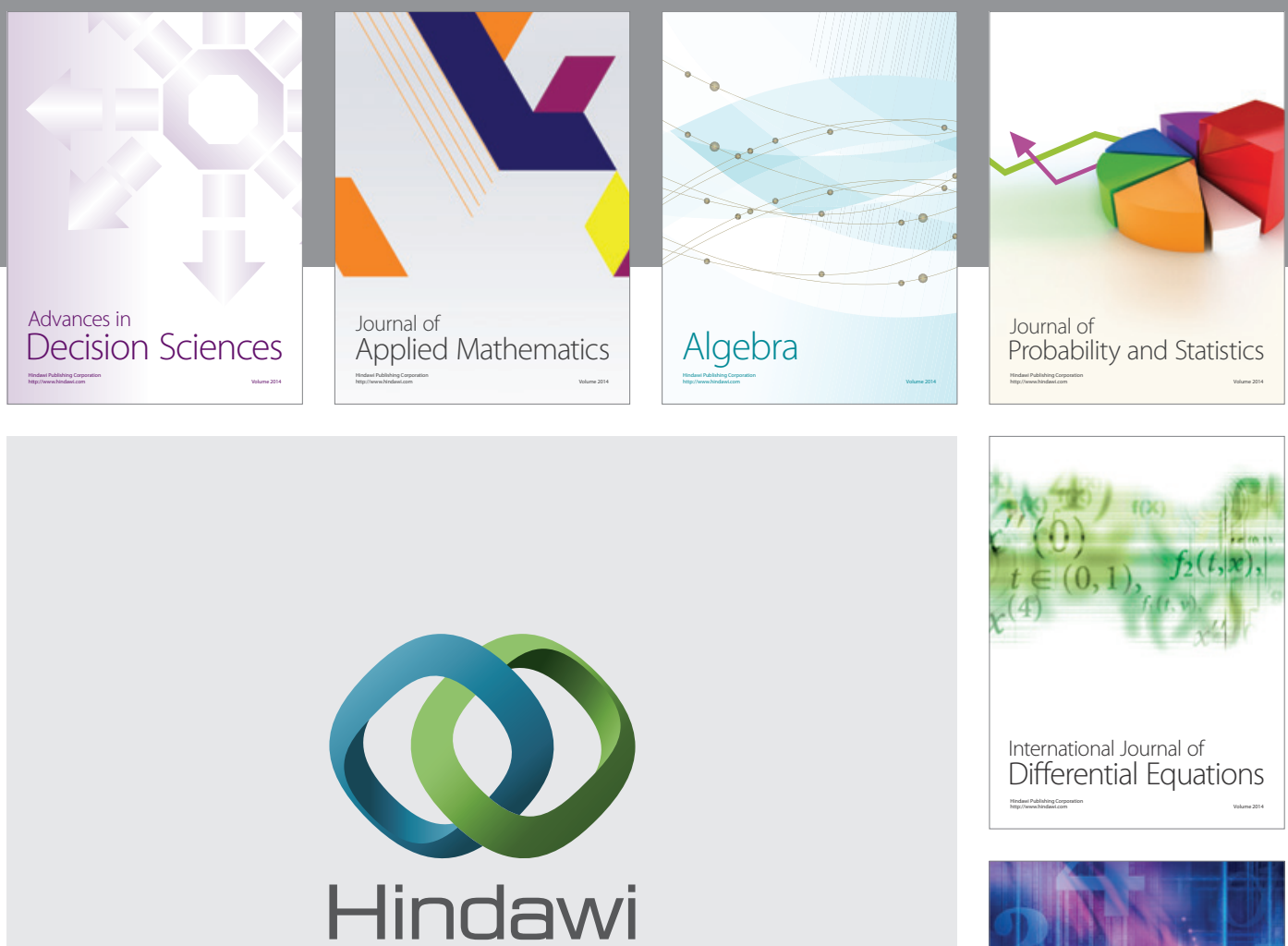

Submit your manuscripts at http://www.hindawi.com
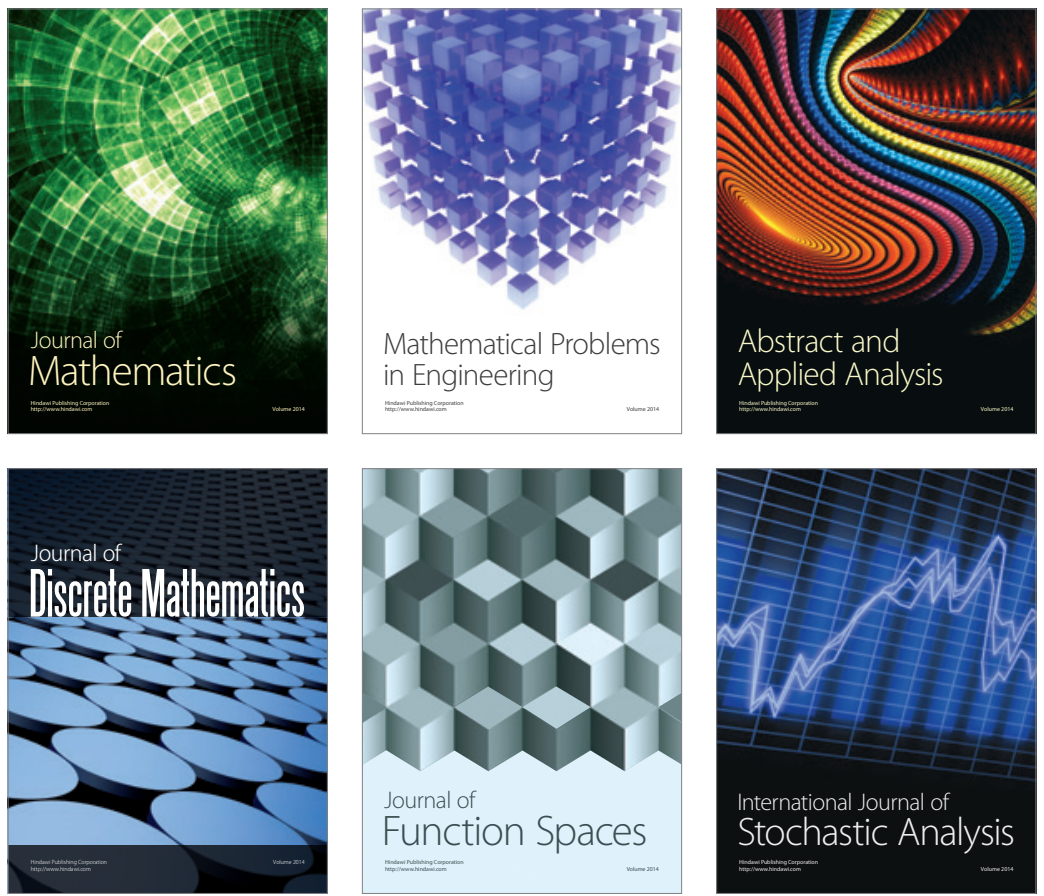

Journal of

Function Spaces

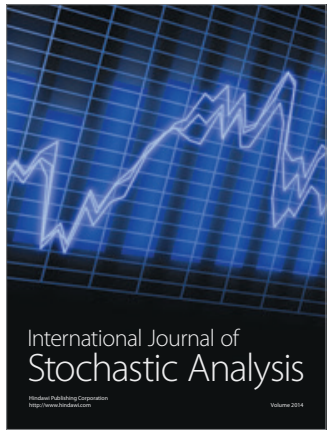

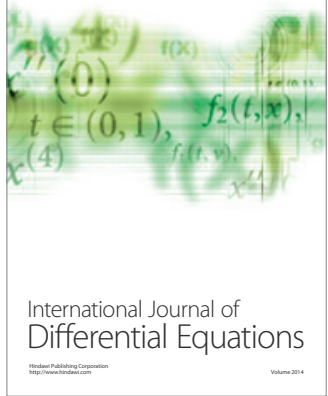
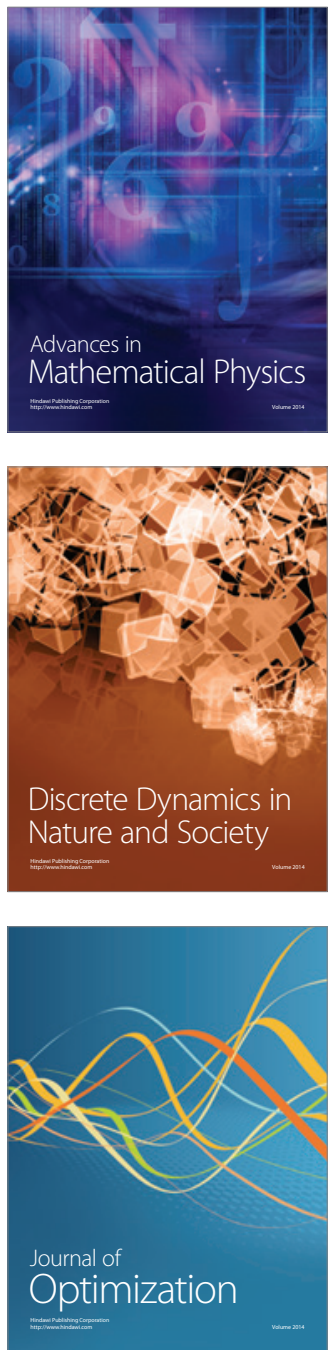